\title{
Entornos virtuales de aprendizaje para la enseñanza de inglés global
}

\author{
Miguel Ángel Candel-Mora ${ }^{\mathrm{a}}, \mathbf{M}^{\mathrm{a}}$ Luisa Carrió-Pastor ${ }^{\mathrm{b}}$ y Ricardo Casañ Pitarch \\ Departamento de Lingüística Aplicada, Universitat Politècnica de València \\ amcandel@upv.es blcarrio@upv.es y $\underline{\text { cricapi@upv.es. }}$
}

\section{\$EWWDWW}

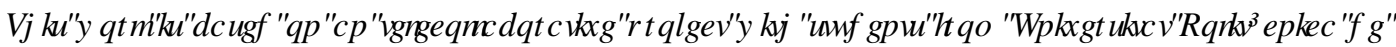

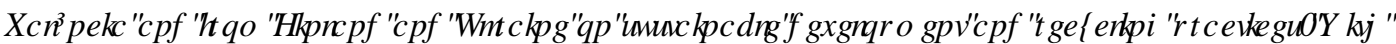

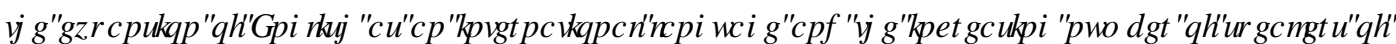

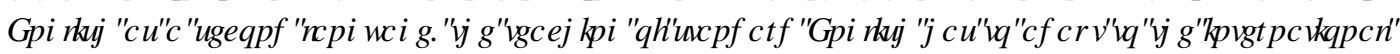

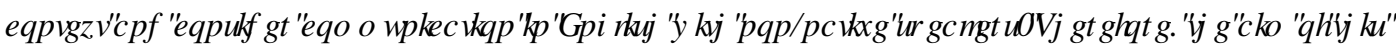

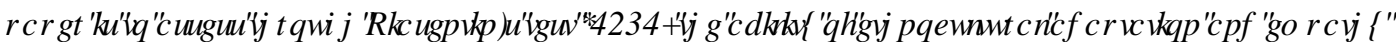

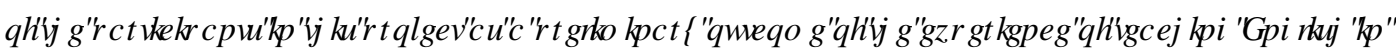

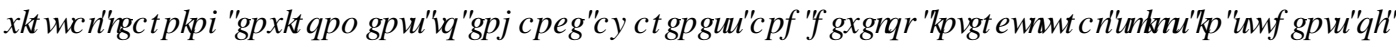

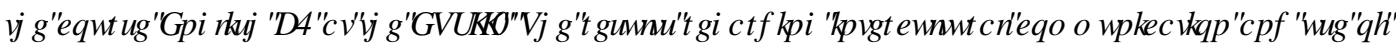

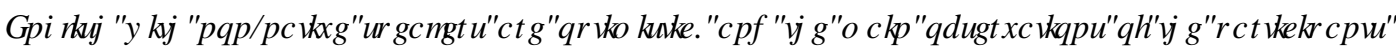

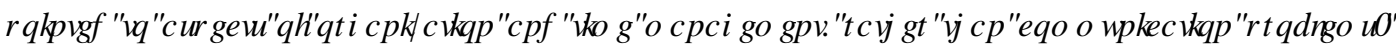

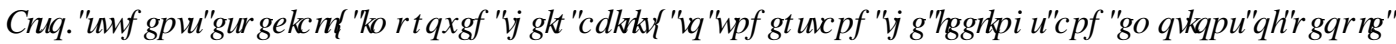
IURP DDGIIHHQWFRXQUU

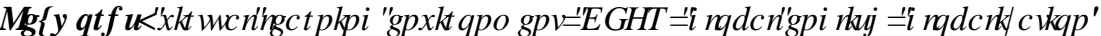

\section{HXP HQ}

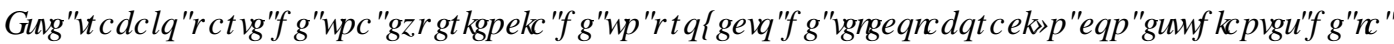

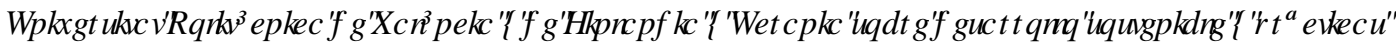

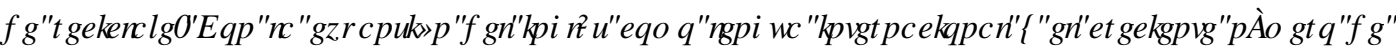

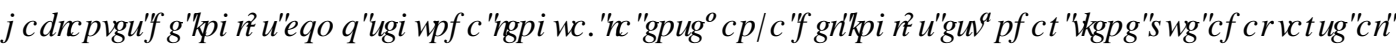

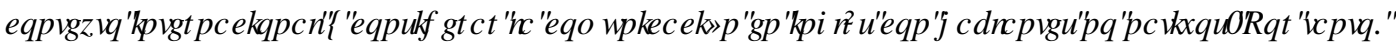

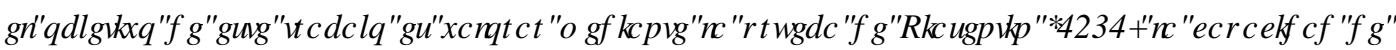

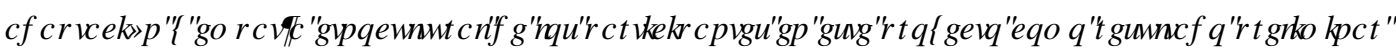

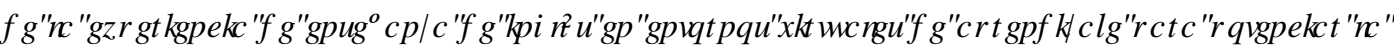

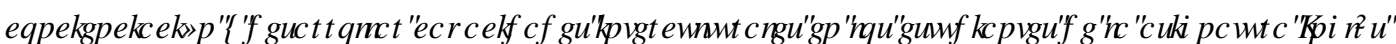

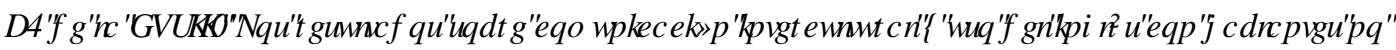

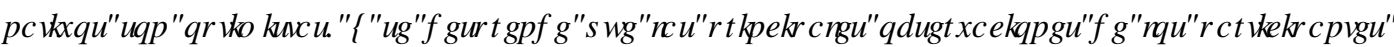

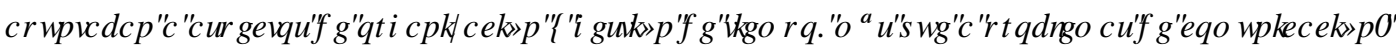

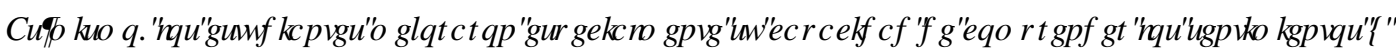

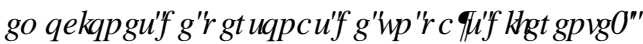

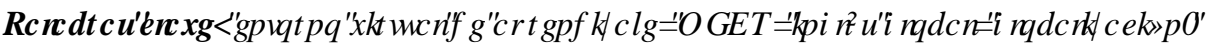




\section{Introducción}

Tras la implantación de los planes de estudios adaptados al plan de Bolonia y el enfoque de competencias en la enseñanza universitaria, y, en consecuencia, la adaptación a las nuevas necesidades y demandas de la sociedad, la asignatura Inglés B2 de la Escuela Técnica Superior de Ingeniería Industrial (ETSII) de la Universitat Politécnica de València también se ha adecuado a este contexto y ha pasado de una enseñanza de inglés para fines específicos en función de la especialidad del grado, a unos contenidos más orientados al entorno académico y profesional actual. Por tanto, se ha realizado una transición desde una enseñanza centrada en los lenguajes de especialidad de cada una de las titulaciones ofertadas (organización industrial, tecnologías industriales, ingeniería química, energía o ingeniería biomédica), a centrarse en unos contenidos centrados en las necesidades y habilidades en lengua inglesa que los egresados en ingeniería pueden necesitar en su futuro entorno académico o profesional.

El temario de la asignatura se estructura alrededor de tres bloques temáticos correspondientes a las principales áreas en las que los ingenieros industriales puede llevar a cabo su desarrollo profesional como son el entorno empresarial, la investigación y el desarrollo, y el contexto académico. Todo ello centrado en habilidades de comunicación profesional, la preparación de informes, correspondencia, presentaciones orales o conversaciones en entornos profesionales, sin olvidar la práctica de los aspectos lingüísticos, discursivos y terminológicos característicos de este nivel B2, siguiendo las directrices del Marco Común Europeo de Referencia para las Lenguas (MCER) (Council of Europe, 2001). El desarrollo de las clases se realiza utilizando una metodología activa y comunicativa en la que el alumno va utilizando gradualmente una expresión más compleja y comprende la necesidad de adaptar esa expresión al contexto comunicativo en el que se encuentre.

En línea con las directrices del MCER, el Consejo de Europa publicó en 2018 una actualización de esta herramienta para adaptarse a los cambios en el uso del lenguaje que ha provocado Internet, la globalización empresarial y la movilidad profesional, entre otras cosas. El nivel B2 mantiene como objetivo la independencia del usuario y además de entender y hablar de diversos temas y mantener una conversación con un nativo requiere del usuario salir del ámbito cotidiano y manejarse en un entorno profesional (North et al., 2018). Sin embargo, uno de los principales cambios es la propuesta de modificación de algunos descriptores que hacen referencia a los "hablantes nativos" como patrón de hablante modelo, concepto que ya causó controversia en la redacción de la primera versión del marco común europeo de referencia para las lenguas (North et al., 2018: 19) .

En la actualidad, existen aproximadamente 1.500 millones de personas que se comunican en inglés con fluidez, de los cuales, 400 millones son hablantes nativos (Crystal, 2008), esto implica que las probabilidades de que un usuario utilice la lengua inglesa como medio de comunicación profesional con hablantes nativos es mucho menor que las de hacerlo con un hablante no-nativo. No obstante, en la actualidad, la mayoría de materiales didácticos procede de editoriales británicas o norteamericanas, que hacen énfasis en la versión estándar de las lenguas habladas en esos países como modelo a seguir, pasando por alto que el concepto de inglés internacional, inglés global o inglés como lengua franca se impone cada vez más en diferentes entornos profesionales y académicos.

Con todo ello, y aprovechando la experiencia del Equipo GECOLER de Innovación y Calidad Educativa de la UPV en proyectos de enseñanza virtual o telecolaboración, surge la idea de explotar la actualización de los descriptores del marco común europeo de referencia de las lenguas (North et al., 2018) que hacen énfasis en la comunicación fluida entre hablantes no nativos y los descriptores específicos que hacen referencia a la conversación y la discusión online, con el fin de actualizar la asignatura a la realidad más inmediata de los alumnos. 
En el contexto de la enseñanza de lenguas, el aprendizaje colaborativo en red ha demostrado su contribución al desarrollo de diferentes aspectos del aprendizaje (O’Dowd y Ware, 2009): autonomía del alumno, precisión y fluidez lingüística, conciencia intercultural, habilidades de comunicación intercultural en línea, alfabetización digital y habilidades blandas (soft skills) como habilidades de trabajo en equipo, creatividad, comunicación interpersonal, pensamiento crítico, habilidades sociales, adaptabilidad y automotivación. Todo esto nos lleva a la realización de diferentes experiencias educativas de colaboración en red que incidan en alguno de los aspectos anteriores y al convencimiento de que se puede dotar a la asignatura de Inglés B2 de mejores instrumentos de medición de las competencias establecidas en nuestras guías docentes.

Este trabajo presenta los resultados de unas pruebas preliminares basadas en el test de Piasentin (2012) para valorar la capacidad de adaptación y empatía etnocultural de los participantes que ayude a potenciar la concienciación y desarrollo de capacidades interculturales.

\section{Objetivos}

Por tanto, el objetivo de este trabajo es presentar los primeros resultados obtenidos durante la fase de planificación de una experiencia de enseñanza de inglés en entornos virtuales de aprendizaje con estudiantes hablantes de inglés no nativos, con diferentes acentos, diferentes contextos culturales y diferentes costumbres de comunicación intercultural obtenidos del test de Piasentin (2012).

Este trabajo se ha dividido en tres partes: en primer lugar, se hace un breve recorrido de la literatura sobre la actualización del marco común europeo de referencia para las lenguas (North et al., 2018) en lo relativo a los cambios en el uso del lenguaje originados por Internet, la globalización empresarial y la movilidad profesional, para destacar las claves de la enseñanza del inglés global como factor de competitividad e integración en el entorno profesional. En segundo lugar, se presenta la contribución de los entornos virtuales de aprendizaje como instrumento clave para la enseñanza del inglés global junto con algunos resultados obtenidos de la implementación de una experiencia piloto de telecolaboración.

\section{Desarrollo de la innovación}

Desde su publicación en 2001, el Marco Común Europeo de Referencia para las Lenguas se utiliza como esquema descriptivo para analizar las necesidades, especificar los objetivos de aprendizaje, dar pautas para la creación de materiales docentes y para la evaluación de los resultados del aprendizaje (Little, 2006), con la intención de actuar de "common basis for the elaboration of language syllabuses, curriculum guidelines, examinations, textbooks, etc. across Europe" (Council of Europe, 2001: 1). El MCER también define los descriptores del dominio de la lengua respecto a los niveles comunes de referencia establecidos en el documento, según Little (2006: 167) una de las partes más utilizadas junto con la denominada escala global y listas de autoevaluación. Aunque el grado de aceptación e implementación del MCER se ha realizado gradualmente, el MCER es una de las principales iniciativas en el campo de la lengua del Consejo de Europa, y en la actualidad se considera el instrumento clave para el desarrollo de posteriores políticas de multilingüismo y homologación de estudios de idiomas a nivel europeo.

Las repercusiones de Internet, la globalización industrial y la movilidad académica y profesional, junto con la implantación de políticas de plurilingüismo a nivel europeo, han tenido un importante papel en la evolución de las lenguas y su adaptación al nuevo contexto sociolingüístico del siglo XXI por lo que en 2018 se publicó el \&RP SDQRQ 9ROP Hal MCER (North et al., 2018) con la intención de atender las carencias relativas a la mediación lingüística resultantes del incremento de la diversidad lingüística y cultural de la sociedad actual. 
Para este estudio, resultan especialmente significativas las modificaciones del \&RP SDQRQ9 RQP H(North et al., 2018) a los descriptores de las escalas MCER original que hacen énfasis en lo referente al hablante nativo ideal como punto de referencia para medir la competencia de un usuario o estudiante y los intentos por desarrollar un repertorio lingüístico que incluya todas las habilidades lingüísticas, es decir que se identifique el grado de precisión, propiedad y facilidad con la que el usuario se desenvuelve en la lengua extranjera. Por ejemplo, en el apéndice siete de los cambios a los descriptores del MCER se destaca notablemente la redacción y la eliminación del concepto de hablante nativo, al tiempo que el ejemplo que sigue (Figura 1) sobre la comprensión de la conversación entre hablantes es uno de los muchos cambios que se observan en este nuevo documento. Como se puede observar en el siguiente fragmento, las referencias al hablante nativo como modelo de hablante ideal han sido eliminadas y sustituidas por "hablantes de la lengua de llegada."

\section{UNDERSTANDING CONVERSATION BETWEEN OTHER NATIVE SPEAKERS}

Can keep up with an animated conversation between native speakers of the target language.

Can with some effort catch much of what is said around him/her, but may find it difficult to participate effectively in discussion with several native speakers of the target language who do not modify their language speech in any way

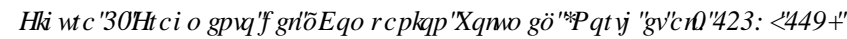

Desde los estudios de Lingüística Aplicada se lleva tiempo investigando el cambio de paradigma de la expansión del inglés como lengua internacional, CQDXDIWDFD, ZRLCH( QJ OKK o inglés global y sus implicaciones pedagógicas (Crystal, 2008; Rose y Galloway, 2019), además de la necesidad de reformular la enseñanza de lenguas para adaptarse al contexto sociolingüístico del siglo XXI.

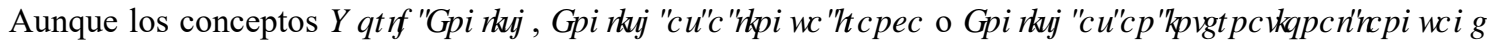
se suelen utilizar como sinónimos, existen ligeras diferencias entre ellos tanto desde el punto de vista de la investigación como de la docencia. Así, según Rose y Galloway (2019), : RLCt( QJ QUK pone el énfasis en los rasgos lingüísticos de las variedades del inglés y sus implicaciones sociolingüísticas. Este enfoque comenzó como disciplina de estudios lingüísticos y sociolingüísticos en los años 70 , aunque centrado en el estudio de las variaciones lingüísticas en el inglés, fundamentalmente de las antiguas colonias británicas (Rose y Galloway, 2019: 5).

( QJCKLVDDOQJ XDIUDQFD explora tanto el uso lingüístico del inglés entre hablantes de diferentes lenguas maternas y las implicaciones sociolingüísticas del uso del inglés como lengua de contacto, así como el estudio del uso del inglés por hablantes nativos de otras lenguas.

Por último, ( QJ CKKDVDQLQULODMRDOQDU XDJHexamina las implicaciones de la expansión del inglés como idioma global centrado fundamentalmente en las implicaciones pedagógicas, y como apuntan Rose and Galloway, (2019: 8) se trata de la versión americana del estudio de inglés como lingua franca que surge la necesidad de estudiar las prácticas lingüísticas en Europa.

Para este trabajo se ha optado por utilizar el término * $\mathbb{R}$ RED( QJ QKK, ya que según diversos autores (Galloway, 2011; Rose y Galloway, 2019) abarca los tres enfoques anteriores y tienen en cuenta la diversidad lingüística, sociolingüística y sociocultural además de la fluidez del uso del inglés y sus usuarios en un entorno globalizado. 


\section{Resultados}

Los primeros cambios en el diseño de la asignatura para abordar la comunicación en inglés con hablantes no nativos se aplicaron en el rediseño del temario de la asignatura y la elección del material docente utilizado en el aula puesto que libro de texto utilizado (Dignen, 2011), además de incluir aspectos teóricos clave de la comunicación intercultural en un entorno profesional y la recreación de situaciones y conversaciones con profesionales de diferentes nacionalidades - en contextos como trabajo en equipo, negociaciones, socializar después del trabajo, realizar presentaciones en público - el material audiovisual que acompaña al manual contiene conversaciones y diálogos en inglés en simulaciones realistas con participantes de diferentes nacionalidades y diferentes acentos: checo, español, japonés, junto hablantes nativos de inglés británico o inglés americano.

Durante las clases en el aula, no se hace tanto hincapié en los contenidos teóricos del manual como en la reflexión general de todos aquellos aspectos culturales y orientaciones que entran en juego durante una interacción con profesionales de diferentes nacionalidades.

Asimismo, la evaluación oral de la asignatura, también contribuye a la consolidación de las claves para la comunicación intercultural ya que los temas sobre los que los alumnos tienen que investigar y preparar una presentación oral, que posteriormente realizarán enfrente del resto de compañeros en el aula, aborda cuestiones como diferencias culturales de diversos países, la forma de realizar negocios a nivel internacional desde diferentes puntos de vista, o cuestiones puramente culturales como el espacio personal, los estereotipos, las situaciones que pueden llevar a un conflicto intercultural, cuestiones de etiqueta o las diferencias culturales en comida, por poner algunos ejemplos.

El uso de entornos virtuales de aprendizaje se aborda habitualmente desde una doble perspectiva: como metodología didáctica y como campo de investigación de los resultados de aprendizaje obtenidos. Esta disciplina se ha desarrollado en los últimos 30 años, al compás de la evolución de la tecnología, y se ha aplicado en campos tan diversos como el marketing, los estudios empresariales o la formación de profesores, aunque la revisión de la bibliografía revela que es en el campo de la comunicación intercultural y la formación en lenguas extranjeras donde parece alcanzar mayores índices, tanto de uso como de explotación de resultados y aprovechamiento (Guth y Helm, 2012; Helm y Guth, 2016; O’Dowd y Ware, 2009).

La tecnología de las plataformas de intercambio virtual o telecolaboración facilita en gran medida el diseño de proyectos y tareas en función de los resultados esperados, especialmente a raíz de la web 2.0 y la participación activa de los usuarios, así como de la mejora de las interfaces y su facilidad de uso. Así pues, del seguimiento de un proyecto de telecolaboración centrado en la enseñanza de lenguas, como es el caso que se presenta en este trabajo, se pueden extraer datos sobre el índice de alfabetización digital, la adquisición de competencias comunicativas interculturales o la dimensión social de la interacción de los participantes, entre otros muchos aspectos.

Este trabajo está basado en una experiencia de aprendizaje colaborativo en entornos virtuales centrado en la enseñanza de lenguas de un proyecto de innovación docente de la UPV en progreso durante el curso académico 2020-21 titulado "Enseñanza de inglés global como lingua franca entre hablantes no nativos en entornos virtuales de aprendizaje como factor de competitividad e integración en el entorno profesional."

La primera fase del proyecto se realizó con alumnos de la Häme University of Applied Sciences (HAMK) de Finlandia y alumnos de la asignatura de inglés B2 de la Escuela Técnica Superior de Ingeniería Industrial de la UPV entre los meses de septiembre y diciembre de 2019. En esta primera fase participaron 22 estudiantes de la UPV y 13 de Finlandia. La segunda fase se realizó con la institución Igor Sikorsky Kyiv 
Polytechnic Institute de Ucrania, con un número similar de participantes de forma piloto, antes de su integración definitiva como parte de una práctica habitual en la asignatura.

Dadas las dificultades surgidas de las diferentes organizaciones de las escuelas, estructuración de los grados, o diferentes metodologías docentes, se optó por elegir un tema de interés común a todos como es la sostenibilidad y concretamente las experiencias de reciclaje en ambos países.

Aunque el proyecto está centrado en la evaluación de la adquisición de la competencia comunicativa en lengua inglesa en general, para este trabajo resulta significativo destacar la exposición de los estudiantes participantes a la comunicación en inglés con hablantes no nativos y, por ende, a la concienciación de un inglés global que será recurrente en su futuro profesional.

Brevemente, el desarrollo del proyecto se puede resumir como sigue:

Se han utilizado las herramientas de Google - Google Classroom, Blogger, Hangouts y Google Drive para facilitar que el acceso y el trabajo de los estudiantes de ambas instituciones esté integrado bajo la misma plataforma, y que por otro lado, resulte familiar para la mayoría de participantes y no requiera demasiada formación adicional.

La participación en el proyecto se plantea como tarea voluntaria para compensar las prácticas informáticas de la asignatura, puesto que el trabajo del proyecto requiere el mismo número de horas y la misma dedicación. Posteriormente, se proporciona a los alumnos las instrucciones detalladas, aunque periódicamente se les envía desde la plataforma recordatorios de las tareas y de las fechas de entrega de las diferentes tareas.

El proyecto está dividido en cinco fases: creación de grupos, formación en terminología y en las características del lenguaje de especialidad del campo de la sostenibilidad, trabajo en grupo, valoración de los participantes del trabajo de los otros grupos, y por último, puesta en común y valoración del proyecto. En todas estas fases, se requiere que los alumnos interactúen entre ellos, puesto que los grupos de trabajo se han diseñado con participantes de ambas instituciones para poner en valor las habilidades de trabajo en equipo y coordinación a distancia y en su segunda lengua.

A lo largo del proyecto, los profesores coordinadores desde ambas instituciones fueron proponiendo diferentes encuestas y tests que, al finalizar el proyecto se procesaron con el fin de obtener la valoración definitiva de los resultados de aprendizaje del proyecto.

Los resultados del progreso en la mejora en habilidades comunicativas en inglés y competencias digitales se han presentado en diferentes publicaciones (Candel-Mora et al., 2020; Casañ-Pitarch et al., 2020; CandelMora y Casañ-Pitarch, 2020), no obstante para este trabajo preliminar destacamos los resultados del progreso de los participantes en la adquisición de la competencia intercultural, clave para la enseñanza de inglés global. 
Para ello se utilizó el cuestionario de Piasentin (2012) con una escala Likert de 5 ítems y preguntas agrupadas en seis bloques (Tabla 1).

\begin{tabular}{|c|c|}
\hline \multicolumn{2}{|c|}{ 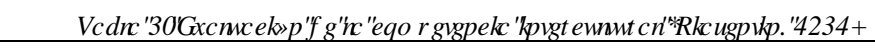 } \\
\hline Bloque 2 & Flexibilidad cognitiva y apertura \\
\hline Bloque 3 & Regulación emocional \\
\hline Bloque 4 & Tolerancia a la incertidumbre \\
\hline Bloque 5 & Autoeficacia \\
\hline Bloque 6 & Empatía etnocultural \\
\hline
\end{tabular}

Los estudiantes realizaron las pruebas previas durante la primera semana del cuatrimestre y las pruebas posteriores después de haber completado el proyecto de trabajo telecolaborativo. Como se muestra en la Tabla 2, el progreso general, considerando la media de los seis bloques, fue del 9,60\% (Tabla 2).

\begin{tabular}{|c|c|c|c|c|}
\hline & Pre-Test & Post-Test & Dif. & $\mathrm{V} \%$ \\
\hline Bloque 1 & 3.81 & 4.23 & 0.42 & 11.02 \\
\hline Bloque 2 & 3.94 & 4.08 & 0.14 & 3.55 \\
\hline Bloque 3 & 3.49 & 3.83 & 0.34 & 9.74 \\
\hline Bloque 4 & 3.48 & 3.87 & 0.39 & 11.21 \\
\hline Bloque 5 & 3.73 & 4.09 & 0.36 & 9.65 \\
\hline Bloque 6 & 3.53 & 3.99 & 0.46 & 13.03 \\
\hline TOTAL & 3.66 & 4.02 & 0.35 & 9.60 \\
\hline
\end{tabular}

Como muestran los resultados, el trabajo con proyectos telecolaborativos en un contexto multinacional es beneficioso para los estudiantes desde una perspectiva intercultural. Las oportunidades que encontraron para practicar sus habilidades lingüísticas durante el desarrollo del proyecto hicieron que el contenido teórico que los participantes habían estudiado en clase pudiera implementarse en un contexto real.

Los resultados muestran que los estudiantes mejoraron su competencia intercultural en aproximadamente un $10 \%$, lo que creemos que es razonable dado el tiempo y el escenario. Con todo, esta oportunidad de trabajo colaborativo virtual fue beneficiosa para los estudiantes de ambas universidades.

\section{Conclusiones}

Tanto desde las instituciones europeas y las políticas de multilingüismo como desde el tejido empresarial se demanda que además del dominio de la lengua, lo que proporciona una ventaja competitiva en el mundo de los negocios son las capacidades lingüísticas y su combinación con capacidades interculturales adicionales (Consejo de Europa, 2007b; Foro empresarial, 2009; Unión Europea, 2019).

Dada la expansión del inglés como lengua internacional y el creciente número de hablantes de inglés como segunda lengua, la enseñanza del inglés estándar también tiene que adaptarse al contexto internacional y tener en cuenta la comunicación en inglés con hablantes no nativos. Asimismo, la multiplicidad de posibilidades de explotación de las experiencias de enseñanza de inglés global en entornos virtuales de aprendizaje hace que esta metodología potencie aspectos de concienciación sobre el uso del inglés en entornos internacionales.

El trabajo con otras competencias incluidas en la guía docente también se ven beneficiadas, por ejemplo, al requerir en todas estas fases que los alumnos interactúen entre ellos, puesto que los grupos de trabajo se 
diseñaron con participantes de ambas instituciones para poner en valor las habilidades de trabajo en equipo y coordinación a distancia y en su segunda lengua.

La respuesta de los alumnos en lo que respecta a la comunicación intercultural y el uso del inglés con hablantes no nativos es positiva, y de la primera implantación del proyecto se desprende que las principales observaciones apuntaban a aspectos de organización y gestión de tiempo, más que a problemas de comunicación.

Los resultados también muestran que los estudiantes mejoraron especialmente su capacidad de comprender los sentimientos y emociones de personas de un país diferente; lo que se conoce en la prueba de Piasentin (2012) como empatía etnocultural. Como se ha sugerido, creemos que la participación en esta experiencia pionera cambió su perspectiva hacia otras realidades, y los participantes rompieron con ciertos prejuicios culturales.

Entre las limitaciones de la investigación, consideramos que la incorporación de nuevas instituciones de otros países podría conducir a la mejora de los presentes resultados. Si los alumnos evitaran el contacto con estudiantes de la misma institución y que hablan el mismo idioma, su grado de inmersión en un contexto multilingüe y multicultural sería mayor. En segundo lugar, implementar este proyecto durante un periodo más largo también sería beneficioso; desafortunadamente, las dos instituciones tenían calendarios académicos diferentes y esto hizo que la duración de este proyecto fuera más corta. A pesar de estas limitaciones, nuestros estudiantes se mostraron satisfechos con la realización de estos proyectos según los resultados de la encuesta de satisfacción final realizada al término del semestre.

Esta experiencia se ha pilotado con estudiantes de la asignatura de inglés B2 de ingeniería industrial, no obstante, dada la implantación generalizada de programas con docencia en inglés en la universidad española, las pautas de planificación y desarrollo de este proyecto serían fácilmente extrapolables a otras asignaturas y titulaciones. Especialmente en el contexto de la pandemia del COVID-19 y tras estos últimos meses de aplicación de métodos de docencia híbrida en el que la gran mayoría de intercambios académicos se han cancelado por la situación sanitaria y los estudiantes han realizado el seguimiento de las asignaturas en la modalidad online.

\section{Agradecimientos}

Este trabajo es uno de los resultados del proyecto de innovación y mejora educativa "PIME/20-21/202 Enseñanza de inglés global como lingua franca entre hablantes no nativos en entornos virtuales de aprendizaje como factor de competitividad e integración en el entorno profesional" financiado por el Vicerrectorado de Estudios, Calidad y Acreditación de la Universitat Poliltècnica de València: Convocatoria Aprendizaje + Docencia 2020. 


\section{Referencias}

CANDEl Mora, M. Á., CARrió PAStor, M. L., y CASAÑ-PitARCh, R. (2020). "Potencial de investigación del aprendizaje colaborativo de inglés profesional en entornos virtuales". En Roig Vila, R. / DIRFHQFDHQ

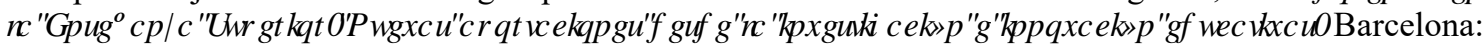
Editorial Octaedro. 1121-1129.

CAndel-Mora, Miguel Ángel; Casañ-Pitarch, Ricardo. (2020). "Desarrollo de destrezas profesionales, lengua extranjera y competencia digital en entornos de aprendizaje virtual". En Polyakova, O. ,QQRYDUMH3 UFUFHIIQ3 XUQDQJ XDOX GXFDMRQ Valencia: Tirant lo Blanch. 92 - 108.

CASAÑ-Pitarch, R., CANDEl-Mora, M. Á., CARrió-Pastor, M. L., Demydenko, O., Y TiKan, I. (2020). "Enhancing language and cross-cultural competence through telecollaboration". \$ GDDFHEt ( GXFDURQ 7(16), 78-87.

CONSEJO DE EUROPA. (2007) "Effects on the European Economy of Shortages of Foreign Language Skills in Enterprise" <https://ec.europa.eu/growth/content/effects-european-economy-shortages-foreignlanguage-skills-enterprise-0 $\mathbf{h r}>$ [Consulta: 25 de enero de 2020]

CONSEJO DE EUROPA. (2007b) "El inglés global no basta para los negocios globales»: conferencia en Bruselas sobre capacidades lingüísticas y competitividad. Comunicado de prensa 21 de septiembre de 2007. $<$ https://ec.europa.eu/commission/presscorner/detail/es/IP_07_1368> [Consulta: 25 de enero de 2020]

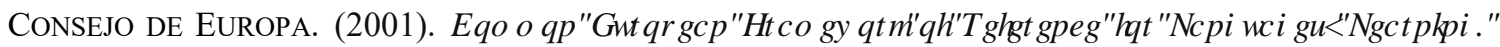
UAFKIQJ $\square$ DMMP HQWCambridge: Cambridge University Press.

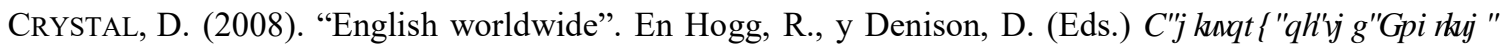
(DQJ XDJH Cambridge: Cambridge University Press. 420-439.

CRYSTAL, D. (1997). ( QJ CKKDWD* REDDO DQJ XDJ H Cambridge: Cambridge University Press.

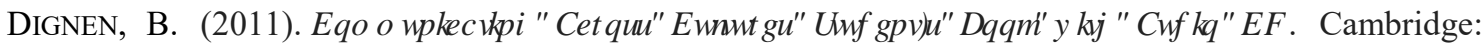
Cambridge University Press.

FORO EMPRESARIAL. (2009). "Las lenguas facilitan los negocios. Las empresas con idiomas rinden más: recomendaciones del Foro Empresarial sobre Multilingüismo creado por la Comisión Europea" $<$ https://op.europa.eu/lv/publication-detail/-/publication/d5ee6ef0-986c-49e6-b8ac-

71da8401efc6/language-es $>$ [Consulta: 25 de enero de 2020]

GUTH, S. Y HELM, F. (2012) "Developing multiliteracies in ELT through telecollaboration”, ( / 7ロ-RXLQDO 66(1): $42-51$.

Helm, F. y Guth, S. (2016). "Telecollaboration and language learning”, En F. Farr y L. Murray. 7KH 5RXIOGJH+ DQCERRNRIV DQJ XDJ HV HDQQQJ DDQCA7HFKQRQRJ . Capítulo 17. New York: Routledge.

LitTLE, D. (2006). "The Common European Framework of Reference for Languages: Content, purpose, origin, reception and impact". / DQJ XDJ H7HDFKQJ, 39. 167-190.

NORTH B., SZABO T., GOODIER T. AND PICCARDO, E. (2018) \&RP PRQ( XLRSHDQ) UP HZRUNRIL5HHHQFH

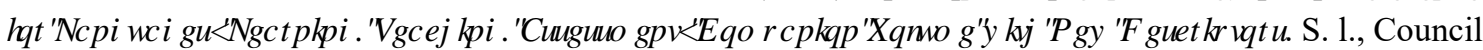
of Europe, <https://rm.coe.int/cefr-companion-volume-with-new-descriptors-2018/1680787989> [Consulta: 25 de enero de 2020]. 
O'DOWD, R Y WARE, P. (2009). “Critical issues in telecollaborative task design”. \&RP SXUAU \$WWHG / DQJ XDJ HV HDQQUТ 22:2, 173-188.

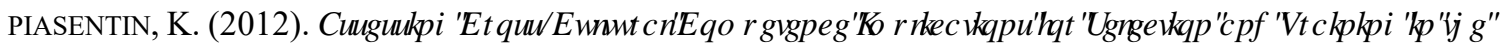
\&DQDCIDQ ) RLFH. Organizational Behaviour Group Socio-Cognitive Systems Section. $<$ https://cradpdf.drdc-rddc.gc.ca/PDFS/unc140/p538194_A1b.pdf $>$ [Consulta: 25 de enero de 2020]

Rose, H., \& Galloway, N. (2019). Global Englishes for Language Teaching. Cambridge: Cambridge University Press.

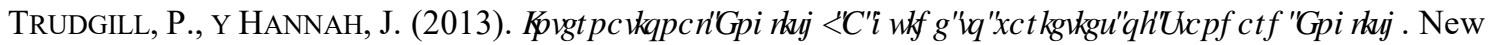
York: Routledge.

UNIÓN EUROPEA. (2019). RECOMENDACIÓN DEL CONSEJO de 22 de mayo de 2019 relativa a un enfoque global de la enseñanza y el aprendizaje de idiomas. Diario Oficial de la Unión Europea $<$ https://eur-lex.europa.eu/legal-content/ES/TXT/PDF/?uri=CELEX:32019H0605(02)\&from=EN> [Consulta: 25 de enero de 2020] 